\title{
Collectif 6t, 2013, Les usages de la mobilité. Pour une
} ingénierie des modes de vie

Paris, Éditions Loco, 168 p.

\section{Julien Aimé}

\section{OpenEdition \\ Journals}

Electronic version

URL: http://journals.openedition.org/rge/5359

DOI: $10.4000 /$ rge.5359

ISSN: 2108-6478

Publisher

Association des géographes de l'Est

Printed version

Date of publication: 30 December 2014

ISSN: 0035-3213

\section{Electronic reference}

Julien Aimé, "Collectif 6t, 2013, Les usages de la mobilité. Pour une ingénierie des modes de vie », Revue Géographique de l'Est [Online], vol. 54 / n³-4 | 2014, Online since 28 February 2015, connection on 25 September 2020. URL : http://journals.openedition.org/rge/5359; DOI : https://doi.org/10.4000/rge. 5359

This text was automatically generated on 25 September 2020 .

Tous droits réservés 


\title{
Collectif 6t, 2013, Les usages de la mobilité. Pour une ingénierie des modes de vie
}

Paris, Éditions Loco, 168 p.

\author{
Julien Aimé
}

\section{REFERENCES}

Collectif 6t, 2013, Les usages de la mobilité. Pour une ingénierie des modes de vie, Paris, Éditions Loco, 168 p.

1 Invitant à s'intéresser aux modes de vie des individus dans leur mobilité, le collectif parisien 6t, bureau d'études composé de chercheurs spécialistes en mobilité, jette ici les bases d'une véritable ingénierie. L'ouvrage, loin d'être uniquement théorique, s'attache à proposer une véritable méthode appliquée de haut niveau pour toute personne souhaitant penser ou «faire » la ville. Sa particularité réside en au moins trois points. L'objet, tout d'abord, semble avoir été minutieusement réfléchi : la maison d'édition Loco soigne le graphisme et la présentation de l'ouvrage, adopte un code couleur pour limiter les parties, propose une typographie particulière et utilise des matériaux de qualité. Ensuite, il s'agit d'un ouvrage écrit directement par ce collectif composé de sociologues, de géographes, d'urbanistes, de mathématiciens, d'économistes, d'architectes et de politistes lors de réunions et séminaires communs. Enfin, l'ensemble du livre s'appuie exclusivement sur les dix années de recherches antérieures de 6t. Ici en effet, nul état de l'art, bibliographie ou revue de la question, il s'agit uniquement de résultats d'études concrets pertinents pour une mise en application sur le terrain.

2 Le collectif propose une « exploration thématique de questionnements prospectifs sur l'avenir de la mobilité urbaine" qu'il décline en 5 chapitres, tous composés (à l'exception du dernier) de 3 parties identiques : « Enjeu », « Postulat » et « Approche ». 
Le sixième et dernier chapitre présente 10 thèses provenant des 5 précédents pour mettre en lumière les leviers d'action permettant la réalisation de cette ingénierie.

3 Le premier chapitre met en exergue les transformations majeures des sociétés industrielles en matière de mobilité, celle-ci étant beaucoup moins contrainte et offrant à l'individu la possibilité de se construire en fonction de cette nouvelle donne sociétale. Les auteurs proposent d'appréhender la mobilité à partir de trois dimensions d'analyse. Le champ des possibles (1), autrement dit tout ce qui joue un rôle sur la mobilité (infrastructures, marchés de l'emploi, politiques d'aide à la mobilité, réseaux disponibles, configurations spatiales, etc.), les aptitudes à se mouvoir (2) qui renvoient aux facteurs permettant de mobiliser et d'utiliser le champ des possibles, et les déplacements «objectifs» (3) des biens et des personnes mais aussi des informations. Prendre conscience de ces différentes dimensions permettrait de dépasser l'approche mécaniste offre/demande pour offrir une véritable réflexion sur les manières d'agir, de dire et de penser des individus ainsi que sur la séduction comme levier de changement des pratiques.

4 Dans un second chapitre, le collectif s'intéresse à l'usage de la voiture et aux différentes alternatives à celle-ci. Bien loin d'être abandonnée, cette dernière est de plus en plus disqualifiée grâce notamment à l'intériorisation progressive des discours écologiques ou éco-responsables. Pourtant, et malgré une offre multimodale quasi-pléthorique (autopartage, covoiturage, transports en communs, taxis, aménagement de pistes cyclables etc.), les auteurs remarquent que les pratiques sont encore exclusivement monomodales, c'est-à-dire que les acteurs continuent de privilégier un seul mode de transport dans leur mobilité quotidienne. Ils appellent donc à développer le « réflexe multimodal " à travers «l'éducation» aux mobilités alternatives dans l'objectif d'optimiser les offres multimodales et alternatives en vue de les intégrer dans un bouquet de services permettant à chaque citoyen d'en tirer profit. Cela permettrait la constitution d'un « cocktail mobilité » (autrement dit l'addition des différents modes de transports potentiellement mobilisables par les individus) suffisamment efficace et pertinent pour changer les habitudes traditionnelles d'utilisation et de possession de l'automobile.

5 Le droit à la mobilité et les effets paradoxaux de la régulation automobile sont au centre du troisième chapitre de l'ouvrage. En narrant l'histoire des politiques de déplacements urbains, les auteurs éclairent les relations entre législations locales et pratiques sociales urbaines. Ils montrent par exemple de quelle manière les politiques de stationnement entraînent des externalités négatives à l'opposé des objectifs fixés par les décideurs publics (relégation spatiale des populations les plus pauvres, discrimination des pendulaires, gentrification des centres-villes, désertion des espaces commerçants hyper-urbains, augmentation de la pollution, etc.). La séduction par l'offre, par la qualité des espaces publics ou encore par un partage de voirie pertinent permettrait de rendre plus attractifs et désirables les modes de transports alternatifs. Selon $6 t$, il conviendrait de réhabiliter la pratique automobile en l'intégrant dans l'offre de transport plutôt qu'en la diabolisant.

6 Les auteurs, dans un quatrième chapitre, abordent la notion d'innovation sociale comme permettant, potentiellement, de répondre aux défis sociaux lancés par l'émergence progressive d'une mobilité plus libre que les politiques spécialisées doivent solutionner. Ces défis sont d'ordre économique (crise économique, augmentation du prix du pétrole, baisse du pouvoir d'achat, etc.), social (inégalité d'accès aux différentes 
formes de mobilité, relégation sociale et spatiale de certaines catégories sociales etc.) et environnemental. Le collectif $6 t$ voit dans l'innovation un moyen de dépasser les différents problèmes bien qu'il faille procéder, encore une fois, avec finesse pour permettre l'effervescence citoyenne dans leur production. Pour le collectif, les technologies de l'information et de la communication, autrement dit l'internet, le téléphone portable et l'open data, sont tout à fait à même d'accompagner et d'aider à l'organisation de ces nouvelles formes de mobilité. Les auteurs présentent également les différents biais qui congestionnent la production de l'innovation sociale tant d'un point de vue local que global (marges de manœuvre trop faibles pour les associations et personnes désireuses d'innover, subsidiarité trop faible...). Il convient alors de libérer la création et l'émulation citoyenne en coordonnant les projets, en les aidant à passer de l'associatif à l'entreprenariat et en formant les individus à la compréhension des outils qu'ils ont en leur possession.

7 Le cinquième chapitre s'ouvre sur une question centrale relative aux politiques de l'aménagement urbain : comment concilier territoire vécu et périmètres institutionnels pour rendre pertinente l'offre de mobilité ? En effet, la ville n'est pas (plus) vécue de manière linéaire et établie, dans le sens ou les trajectoires quotidiennes ne sont plus dirigées de manière unilatérale. Les auteurs proposent donc de rencontrer les individus vivant les territoires en fonction de leur statut (interroger des jeunes, des travailleurs, des habitants, des touristes etc.) et suggèrent d'établir des projets qui intègrent vraiment la population comme "maître d'usage » et "coproducteur " de l'action publique. Ainsi, la mise en place d'ateliers de quartier ou de modules de démocratie participative permettent de rendre plus viable et raisonnée l'offre de mobilité à destination des populations. Mais c'est aussi la rencontre des acteurs-clés, parfois institutionnels, qui peut permettre d'atteindre des populations un peu plus marginalisées, qui conscientisent moins leur rapport à la mobilité ou qui sont moins enclines à en rendre compte. C'est à partir d'un panel de formules participatives (tables rondes, atelier de travail, concertation productives...) assez détaillé que les auteurs proposent d'améliorer la compréhension du rapport entre territoire et périmètres.

8 Le dernier chapitre, enfin, fait office de conclusion. Les auteurs y présentent dix thèses permettant de donner corps à ce qu'ils appellent « l'ingénierie de la mobilité pensée à partir des modes de vie ». Chaque thèse reprend, peu ou prou, le développement des chapitres précédents. Elles proposent, entre autres, de s'intéresser aux cadres de vie des individus pour proposer une offre de transport plus en adéquation avec les pratiques sociales et d'axer les politiques sur l'offre multimodale, alternative à l'autosolisme (pratique individuelle de l'automobile). Elles exhortent les penseurs de la ville à s'intéresser à l'offre de mobilité par le biais du plaisir, de la séduction et de l'éducation plutôt que par l'aspect fonctionnel (rapport coût/temps de déplacement), sans jamais oublier que c'est l'individu individualisé qui est au cœur de ces pratiques.

9 Cet ouvrage représente donc assez bien le lien étroit entre recherche publique et expertise privée, et montre avec pertinence comment les différentes disciplines peuvent travailler de concert pour proposer des solutions adaptées aux problématiques très récentes de la mobilité. Le livre étonne par sa mise en page, sa forme et sa facilité de lecture, ce qui en fait un ouvrage accessible tant aux étudiants qu'aux professionnels et spécialistes de la question. Si on peut toujours interroger le fait de jouer in fine le jeu de la rationalisation économique de la recherche en sciences sociales, il reste que ce qui semble guider l'ouvrage, c'est avant tout l'objectif de satisfaire le «bien commun » et 
de permettre de décloisonner le rapport entre les politiques et les individus qui ne les choisissent pas.

\section{AUTHOR}

\section{JULIEN AIMÉ}

Doctorant en Sociologie à l'Université de Lorraine - Laboratoire 2L2S - 23, boulevard Albert $1^{\text {er }}$ BP 339754015 Nancy CEDEX 